\title{
A tracer of organic matter of prebiotic interest in space, made from UV and thermal processing of ice mantles
}

\author{
G. M. Muñoz Caro $^{1}$ and E. Dartois ${ }^{2}$
}

\author{
${ }^{1}$ Centro de Astrobiología (CSIC-INTA), Carretera de Ajalvir, km 4, Torrejón de Ardoz, 28850 Madrid, Spain \\ e-mail: munozcg@inta.es \\ 2 Institut d'Astrophysique Spatiale, UMR 8617, Bât. 121, Université Paris XI, 91405 Orsay, France
}

Received 2 June 2008 / Accepted 17 October 2008

\section{ABSTRACT}

\begin{abstract}
Aims. Our main goal was to identify an infrared spectral feature that can serve as a tracer of $\mathrm{O}$ and $\mathrm{N}$-rich solid organic matter in space. Such material is expected to result from UV-irradiation and sublimation of icy grain mantles in certain environments, including hot cores and regions around YSOs. Our analysis of the $3.4 \mu \mathrm{m}$ feature, present in the spectra of organic refractory samples made from UV-irradiation of interstellar/circumstellar ice analogs, indicates that the $3.4 \mu \mathrm{m}$ band is a potential tracer of $\mathrm{O}$ and $\mathrm{N}$-rich solid organic matter of prebiotic interest in space.

Methods. We carried out simulation experiments of UV-photoprocessing and sublimation of ice mantles in dense clouds and circumstellar regions leading to the formation of organic refractory residues under different conditions. The analysis of the deposited ice and the organic residue products was made in situ by infrared spectroscopy. Spectral comparison of these residues to molecular standards for compositional characterization was performed.

Results. For ice mixtures of different composition, UV-photon dose and frequency, the $3.4 \mu \mathrm{m}$ feature of the organic residue product at room temperature shows a broad double-peaked profile with subfeatures at $\sim 2926 \mathrm{~cm}^{-1}(3.42 \mu \mathrm{m})$ and $2876 \mathrm{~cm}^{-1}(3.48 \mu \mathrm{m})$, mainly due to $\mathrm{CH}_{2}$ groups adjacent to $\mathrm{OH}$ groups. This feature profile was not reproduced in similar UV-irradiation experiments using non-realistic analogs of interstellar ice mantles. In the astrophysical context, this infrared feature is thus expected to be characteristic of the products resulting from ice UV-irradiation and sublimation; it is fully distinct from the $3.4 \mu \mathrm{m}$ feature observed in the diffuse interstellar medium, which is attributed to hydrogenated amorphous carbon.

Conclusions. A $3.4 \mu \mathrm{m}$ band with a similar broad double-peaked profile tracing organic refractory matter of prebiotic interest could be searched for in regions where icy grain mantles were exposed to UV-irradiation prior to sublimation, like hot cores and regions around YSOs. This band may also be present in some cometary nuclei that preserve such grains, provided that the annealing temperatures experienced were not higher than $\sim 400 \mathrm{~K}$.
\end{abstract}

Key words. ISM: molecules - methods: laboratory - ultraviolet: ISM - dust, extinction - infrared: ISM

\section{Introduction}

Icy grain mantles, composed mainly of $\mathrm{H}_{2} \mathrm{O}$ and species like $\mathrm{CH}_{3} \mathrm{OH}, \mathrm{CO}, \mathrm{CO}_{2}$ and $\mathrm{NH}_{3}$ (hydrated or as $\mathrm{NH}_{4}^{+}$), are observed in dense interstellar clouds and circumstellar regions (Gerakines et al. 1999; Ehrenfreund et al. 1999; Dartois et al. 1999; Gibb et al. 2001; Schutte \& Khanna 2003; Thi et al. 2002; Pontoppidan et al. 2005). They are submitted to UV irradiation in those environments. Shen et al. (2004) show that the energy input by the cosmic-ray induced ultraviolet field is almost one order of magnitude greater than the direct energy input by cosmic-ray particles. Ice mantles in hot cores and regions around YSOs are processed by the UV flux emitted by the central object and that impinging from the surrounding interstellar medium. In addition, the temperature increases close to the central object, leading to sublimation of the ice mantles, see Muñoz Caro \& Schutte (2003) for a short summary.

Experimental simulations of ice photo- and thermal processing in the interstellar/circumstellar medium lead to the formation of an organic refractory residue that is observed after warm-up to room temperature. Abundant species detected in these residues are hexamethylenetetramine $\left[\left(\mathrm{CH}_{2}\right)_{6} \mathrm{~N}_{4}\right]$, ammonium salts of carboxylic acids $\left[\left(\mathrm{R}-\mathrm{COO}^{-}\right)\left(\mathrm{NH}_{4}^{+}\right)\right]$, amides $\left[\mathrm{H}_{2} \mathrm{NC}(=\mathrm{O})-\mathrm{R}\right]$, esters $\left[\mathrm{R}-\mathrm{C}(=\mathrm{O})-\mathrm{O}-\mathrm{R}^{\prime}\right]$, and species based on polyoxymethylene $\left[\left(-\mathrm{CH}_{2} \mathrm{O}-\right)_{n}\right]$. Other residue components of clear prebiotic interest are amino acids or their precursors and $\mathrm{N}$-heterocyclic molecules, both present with an abundance of the order of $\sim 1 \%$ by number of molecules (Agarwal et al. 1985; Briggs et al. 1992; Bernstein et al. 1995, 2002; Muñoz Caro et al. 2002; Muñoz Caro \& Schutte 2003; Meierhenrich et al. 2005; Nuevo et al. 2006).

Irradiation of ice mantles by UV photons should be considered a common phenomenon in circumstellar regions and dense clouds. There is, however, no current evidence for the presence of relatively large organic species, like those made by ice photoprocessing in the laboratory, embedded in the ice matrix (along with the common ice components mentioned above, the largest species detected in the infrared spectra of ice mantles are $\mathrm{CH}_{3} \mathrm{CHO}, \mathrm{HCOOH}, \mathrm{OCN}^{-}$, and $\mathrm{OCS}$ ). This could be due to the broad features of abundant volatile ices which hinder the detection of minor organic components (Muñoz Caro \& Schutte 2003).

Here we search for an infrared feature that could serve as a tracer of $\mathrm{O}$ and N-rich solid organic matter of prebiotic interest in space, made by UV-photoprocessing and sublimation of icy grain mantles, in environments such as hot cores and regions around YSOs; our search is based on experimental simulations of ice processing by UV photons, leading to the formation of 
organic refractory residues. Firstly, such tracer of organic matter should preferably be a feature with a characteristic band profile, to ensure that the feature is not confused with other absorption bands in the same spectral region. Secondly, the feature should be present in the spectra of organic refractory residues, made in the laboratory under different but astrophysically relevant conditions; that way, we tried to select a feature that could be observed in a variety of regions where ice mantles are exposed to UV-irradiation followed by sublimation.

It was observed that bands due to functional groups in the spectra of organic refractory residues differ, depending on experimental parameters like the starting ice composition (Muñoz Caro \& Schutte 2003). We found that, among the bands present in the spectra of organic refractory residues, the best candidate as a tracer of organic matter made by ice UV-processing in space was the $3.4 \mu \mathrm{m}$ feature, in the region $3000-2750 \mathrm{~cm}^{-1}$. This feature fulfills the first condition given above, as it displays a characteristic profile composed of two main bands, attributed to $\mathrm{CH}_{2}$ stretching modes (Allamandola et al. 1988). Although the $3.4 \mu \mathrm{m}$ feature of organic refractory residues can overlap with the red wing of the broad absorption band due to the $\mathrm{O}-\mathrm{H}$ stretching in $\mathrm{H}_{2} \mathrm{O}$ ice, or the $\mathrm{C}-\mathrm{H}$ stretching modes in simple ice species like $\mathrm{CH}_{3} \mathrm{OH}$ and $\mathrm{CH}_{4}$, the band profiles are notably different, and after ice sublimation only the $3.4 \mu \mathrm{m}$ feature of the organic refractory residue remains. The second requirement, i.e. whether or not the double-peaked $3.4 \mu \mathrm{m}$ feature is present in the spectra of organic refractory residues made in the laboratory under different astrophysically relevant conditions, is studied here in detail, by varying the composition of the starting ice mixture, UV-irradiation dose, and UV frequency. In addition, the organic components responsible for the infrared $3.4 \mu \mathrm{m}$ feature observed in the organic refractory residue spectra are characterized in this paper.

\section{Experimental}

The experimental setup consists of a high vacuum chamber where a gas mixture is deposited onto a cold finger and UVirradiated. The pressure of the system at room temperature is $P \approx 1 \times 10^{-7}$ torr. At the cold finger the temperature is $T \approx$ $12 \mathrm{~K}$. The cold finger consists of a sample holder, in which an IR-transparent CsI window is mounted.

The gas mixture is prepared by filling a bulb with different gases while the partial vapor pressures are monitored. The vacuum pressure of the gas line is $P \approx 10^{-5}$ torr. The typical rate of deposition was $2 \times 10^{15}$ molecules $\mathrm{cm}^{-2} \mathrm{~s}^{-1}$, and the deposition time was $13 \mathrm{~h}$. During the deposition, the ice layer is simultaneously UV-irradiated with a microwave stimulated hydrogen flow discharge lamp (output $\approx 1.5 \times 10^{15}$ photons $\mathrm{s}^{-1}$, Weber \& Greenberg $\left.1985 ; E_{\text {photon }}=7.3-10.5 \mathrm{eV}\right)$, separated from the vacuum chamber by a $\mathrm{MgF}_{2}$ window. The main peak emission is at Lyman- $\alpha(121 \mathrm{~nm}$ or $10.2 \mathrm{eV})$ for a hydrogen pressure $P_{\mathrm{H}}=0.5$ torr. The UV photon flux at the position of the sample is $F_{\mathrm{UV}} \approx 5 \times 10^{14}$ photon $\mathrm{cm}^{-2} \mathrm{~s}^{-1}$, resulting in an average dose of $\sim 0.25 \mathrm{UV}$ photon $\mathrm{molec}^{-1}$. The long simultaneous deposition and irradiation time of $13 \mathrm{~h}$, and the high photon dose of $\sim 0.25 \mathrm{UV}$ photon molec $^{-1}$, served to obtain a sufficient amount of organic refractory residue to allow infrared spectroscopy (Muñoz Caro \& Schutte 2003; this paper), and characterization by chromatographic techniques (e.g. Muñoz Caro et al. 2004). For one of the experiments a quartz window was placed in the interface between the UV lamp and the high vacuum chamber, replacing the usual $\mathrm{MgF}_{2}$ window; the quartz transmission has a cutoff at about $140 \mathrm{~nm}(8.9 \mathrm{eV})$ giving an output $\approx 4 \times 10^{14}$ photons $\mathrm{s}^{-1}$ (henceforth soft UV spectrum). After irradiation, the system is warmed up gradually by means of a temperature controller. For details on the experimental protocol see Muñoz Caro \& Schutte (2003).

The evolution of the sample during irradiation and warm-up was monitored by Fourier transform infrared, FT-IR, transmission spectroscopy at a resolution of $2 \mathrm{~cm}^{-1}$. For all the experiments, the final spectrum of the organic refractory residue that remains after ice sublimation was taken under high vacuum after 10 hours at room temperature.

\section{Experimental results}

\subsection{The carriers of the $3.4 \mu \mathrm{m}$ feature of organic refractory residues}

Figure 1 shows the aliphatic $\mathrm{C}-\mathrm{H}$ stretching bands $(3.4 \mu \mathrm{m}$ feature) of organic refractory residues made starting from different ice mixtures, UV dose and frequency, see Table 1 adapted from Muñoz Caro \& Schutte (2003). This band falls between 3000 and $2750 \mathrm{~cm}^{-1}$ and generally presents a characteristic broad double-peaked profile in the organic refractory residue spectra. The common peaks at $2926 \mathrm{~cm}^{-1}$ and $2876 \mathrm{~cm}^{-1}$ correspond mainly to the aliphatic $\mathrm{CH}_{2}$ asymmetric and symmetric stretching modes, although we will later show that there is some contribution from $\mathrm{CH}_{3}$ stretchings to these subfeatures. A subfeature peaking around $2790 \mathrm{~cm}^{-1}$, attributed to $\mathrm{CH}_{3}$ groups attached to $\mathrm{N}$, is observed in some of the organic refractory residue spectra of Fig. 1. Hexamethylenetetramine (HMT, $\left.\left[\left(\mathrm{CH}_{2}\right)_{6} \mathrm{~N}_{4}\right]\right)$ is a contributor to this band; it has peaks at 2874 ( $v_{18}$ sym. $\mathrm{CH}_{2}$ stretch), 2924 ( $2 v_{19} \mathrm{CH}_{2}$ deformation), and $2949\left(v_{17}\right.$ asym. $\mathrm{CH}_{2}$ stretch) $\mathrm{cm}^{-1}$ (Bernstein et al. 1994, 1995). The specific HMT fingerprints, however, are two sharp peaks at 1234 and $1007 \mathrm{~cm}^{-1}$ that correspond to the $v_{21}$ and $v_{22} \mathrm{CN}$ stretch. Other HMT features are seen at $1461 \mathrm{~cm}^{-1}$, $1368 \mathrm{~cm}^{-1}, 815 \mathrm{~cm}^{-1}$ and $671 \mathrm{~cm}^{-1}$ (Bernstein et al. 1995). Indeed, the HMT band at $2949 \mathrm{~cm}^{-1}$ is best observed in the $3.4 \mu \mathrm{m}$ spectrum of OR16, the residue with the highest concentration of HMT (see Fig. 1 and Table 1). Nevertheless, the $\mathrm{CH}$ stretching modes of HMT at $3000-2825 \mathrm{~cm}^{-1}$ account only for $\sim 15 \%$ of the $3.4 \mu \mathrm{m}\left(2941 \mathrm{~cm}^{-1}\right)$ feature in residue OR1, our standard residue; that value is inferred from integration of the $3.4 \mu \mathrm{m}\left(2941 \mathrm{~cm}^{-1}\right)$ and $1007 \mathrm{~cm}^{-1}$ features in the spectrum of OR1, and using the absorption ratio between the $3.4 \mu \mathrm{m}$ and the $1007 \mathrm{~cm}^{-1}$ bands in pure HMT from Bernstein et al. (1994). Thus, typically about $85 \%$ of the $3.4 \mu \mathrm{m}$ feature is due to aliphatic carriers different from HMT, which we try to characterize below. To facilitate this, we tried to reproduce the $3.4 \mu \mathrm{m}$ feature of organic refractory residues using ice mixtures with the simplest composition.

From the common molecular components of icy grain mantles in interstellar and circumstellar environments, i.e. $\mathrm{H}_{2} \mathrm{O}$, $\mathrm{CH}_{3} \mathrm{OH}, \mathrm{CO}, \mathrm{CO}_{2}$, and $\mathrm{NH}_{3}$, the only molecule deposited as pure ice that leads to a significant amount of residue after UV-irradiation and warm-up was found to be $\mathrm{CH}_{3} \mathrm{OH}$ (Fig. 2, top panel). This spectrum shows features at 3404 (H-bonded $\mathrm{OH}$ str.), 1712 ( $\mathrm{C}=\mathrm{O}$ str. in saturated aliph. ketones), and $1107 \mathrm{~cm}^{-1}$ (C-O-C asym. str. in aliph. ethers). Bands due to aliphatic $-\mathrm{CH}_{2}$ - and $-\mathrm{CH}_{3}$ groups are observed at 2971 (- $\mathrm{CH}_{3}$ asym. str.), 2935 (- $\mathrm{CH}_{2}$ - asym. str.), 2875 (- $\mathrm{CH}_{3}$ sym. str.), 1455 (- $\mathrm{CH}_{2}$ - bend), and 1376 (aliph. $-\mathrm{CH}_{3}$ sym. bend).

Only when $\mathrm{CH}_{3} \mathrm{OH}$ is co-deposited with $\mathrm{H}_{2} \mathrm{O}$ does the residue obtained from ice irradiation and warm-up show the $3.4 \mu \mathrm{m}$ feature with the broad double-peaked profile of organic 
Table 1. Experimental parameters.

\begin{tabular}{ccccccccc}
\hline \hline $\begin{array}{c}\text { Exp. } \\
\mathrm{OR \#}^{1}\end{array}$ & Comment & $\begin{array}{c}\text { Ice mixture } \\
\mathrm{H}_{2} \mathrm{O}: \mathrm{NH}_{3}: \mathrm{CH}_{3} \mathrm{OH}: \mathrm{CO}: \mathrm{CO}_{2}\end{array}$ & $\begin{array}{c}\text { Dose } \\
\text { photon } \\
\text { molec }\end{array}$ & $\begin{array}{c}\frac{N_{\mathrm{C}} \text { (HMT) }}{N_{\mathrm{C}} \text { (ice) }} \\
(\%)\end{array}$ & $\begin{array}{c}\frac{N_{\mathrm{C}} \text { (c.a.salt) }}{N_{\mathrm{C}} \text { (ice) }} \\
(\%)\end{array}$ & $\begin{array}{c}\frac{N_{\mathrm{C}} \text { (amide) }}{N_{\mathrm{C}} \text { (ice) }} \\
(\%)\end{array}$ & $\begin{array}{c}\frac{N_{\mathrm{C}} \text { (ester) }}{N_{\mathrm{C}} \text { (ice) }} \\
(\%)\end{array}$ & $\begin{array}{c}\frac{N_{\mathrm{C}} \text { (total) }}{N_{\mathrm{C}} \text { (ice) }} \\
(\%)\end{array}$ \\
\hline 1 & standard exp. & $2: 1: 1: 1: 1$ & 0.25 & $6.4 \pm 0.3$ & 1.8 & 0.33 & 0.16 & 8.7 \\
4 & & $2: 1: 1: 1: 1$ & 3.33 & $5.6 \pm 0.1$ & 1.5 & 1.2 & 0.9 & 9.2 \\
5 & & $2: 1: 1: 1: 1$ & 0.031 & $2.4 \pm 0.1$ & 0.3 & 0.04 & 0.10 & 2.8 \\
7 & \multirow{2}{*}{ soft UV spectrum } & $2: 1: 1: 1: 1$ & 0.66 & $7.0 \pm 0.3$ & 0.4 & 0.15 & 0.13 & 7.7 \\
9 & & $0: 1: 1: 0: 0$ & 0.32 & $0.6 \pm 0.1$ & 0.09 & 0.27 & 0.05 & $1.0^{2}(1.5)$ \\
13 & & $2: 1: 1: 0: 0$ & 0.25 & $0.4 \pm 0.2$ & 0.08 & $\leq 0.01$ & $\leq 0.01$ & $0.5^{2}(0.8)$ \\
16 & & $20: 1: 1: 1: 1$ & 0.22 & $15.0 \pm 0.7$ & 0.3 & 0.28 & 0.11 & 15.7 \\
\hline
\end{tabular}

${ }^{1}$ Experiments are named "OR" followed by a number. OR stands for organic refractory residue.

${ }^{2}$ In addition to the listed species, the spectrum shows a large abundance of POM-like species. The values in brackets include the POM abundances assuming the band strength of pure POM $\left(9.7 \times 10^{-18} \mathrm{~cm}(\mathrm{C} \text { atom })^{-1}\right.$ from Schutte et al. 1993).

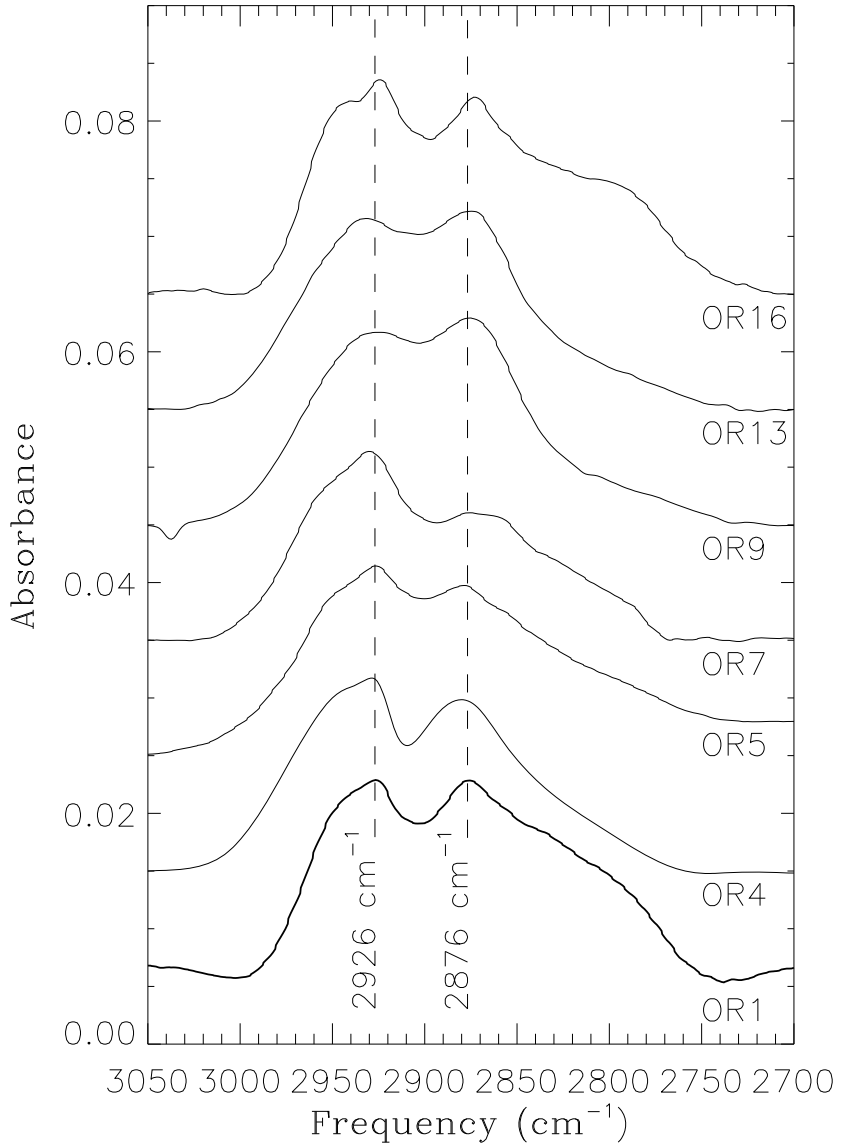

Fig. 1. Organic refractory residue spectra from photoprocessing of different ice mixtures. See Table 1 for experimental parameters of the samples.

refractory residues. This is illustrated in Fig. 2, bottom panel, showing the organic refractory residue spectrum obtained from overnight UV-irradiation of $\mathrm{H}_{2} \mathrm{O}:{ }^{13} \mathrm{CH}_{3} \mathrm{OH}=1: 1$ ice. The $\mathrm{CH}_{2}$ - and $-\mathrm{CH}_{3}$ bendings are seen at 1448 and $1367 \mathrm{~cm}^{-1}$. Other features are the prominent band between $3600-2600 \mathrm{~cm}^{-1}$ (H-bonded $\mathrm{OH}$ str. in polymers, probably due to alcohols and carboxylic acids), $1677 \mathrm{~cm}^{-1}(\mathrm{C}=\mathrm{O}$ str. of carboxylic acid dimers), $1568 \mathrm{~cm}^{-1}$ (unknown), $1207 \mathrm{~cm}^{-1}$ (possibly the $\mathrm{C}-\mathrm{O}-\mathrm{C}$ str. in ethers) and $1029 \mathrm{~cm}^{-1}$ (possibly the $\mathrm{CH}_{2} \mathrm{OH}$ in primary alcohols).

The results from the above experiment show that the broad double-peaked $3.4 \mu \mathrm{m}$ feature of organic refractory residues is
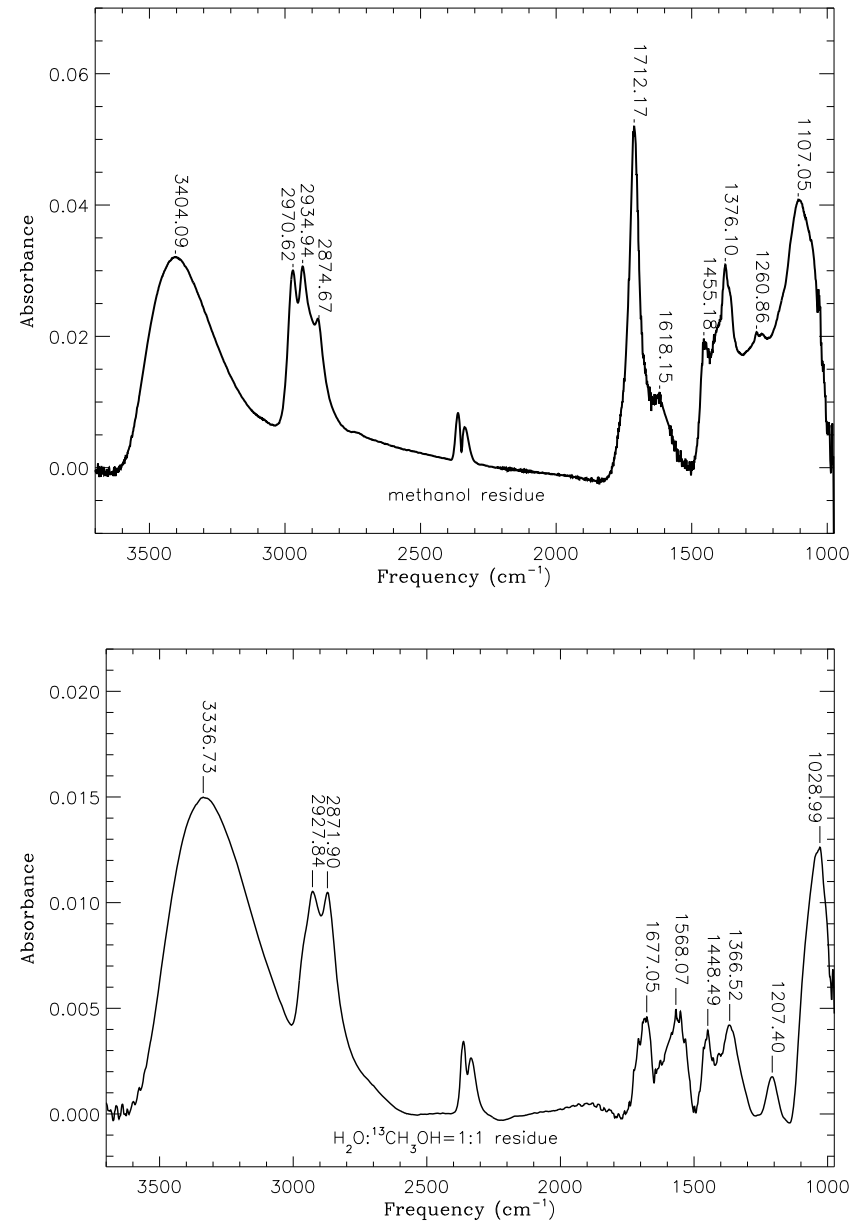

Fig. 2. Top: residue spectrum from UV-irradiation of methanol ice. Bottom: residue spectrum from UV-irradiation of $\mathrm{H}_{2} \mathrm{O}: \mathrm{CH}_{3} \mathrm{OH}=$ 1:1 ice. The band between $2400-2300 \mathrm{~cm}^{-1}$ is $\mathrm{CO}_{2}$ gas absorbing along the beam of the infrared spectrometer.

also obtained by irradiation of ices that do not contain N. That means that HMT, a N-bearing molecule, cannot be present in those organic refractory residues, and yet the spectra of these residues show the characteristic broad double-peaked $3.4 \mu \mathrm{m}$ feature. If $\mathrm{CH}_{3} \mathrm{OH}$ is not a component of the starting ice mixture, the absorbance values of the $3.4 \mu \mathrm{m}$ feature are reduced (Jenniskens et al. 1993), suggesting that $\mathrm{CH}_{3} \mathrm{OH}$, that converts efficiently into formaldehyde $\left(\mathrm{H}_{2} \mathrm{CO}\right)$ upon irradiation initiating chemical reactions is the main precursor of the $3.4 \mu \mathrm{m}$ 
feature carriers. Although $\mathrm{CH}_{3}$ groups are present, as shown by the band at $1367 \mathrm{~cm}^{-1}$ attributed to the $\mathrm{CH}_{3}$ symmetric deformation mode, the carrier species of the $3.4 \mu \mathrm{m}$ feature must have high $-\mathrm{CH}_{2}-/-\mathrm{CH}_{3}$ ratios because the $-\mathrm{CH}_{3}$ asym. str. mode around $2960 \mathrm{~cm}^{-1}$ is relatively weak or not observed compared to the asym. and sym. $-\mathrm{CH}_{2}$ - str. modes observed at $\sim 2926$ and $2876 \mathrm{~cm}^{-1}$, indicating different options: i) the aliphatic chains are long and poorly branched; ii) cyclic or cage molecules with $\mathrm{CH}_{2}$ and no $\mathrm{CH}_{3}$ groups; iii) chains of the type $\mathrm{R}-\left(\mathrm{CH}_{2}\right)_{n}-\mathrm{R}^{\prime}$ where $n$ does not need to be a large number because there are no $-\mathrm{CH}_{3}$ bonds in the molecular structure. These three options are discussed below:

i) This possibility is unlikely because the $\mathrm{O}$ in O-bearing ice species enters the composition of the irradiation products, e.g. the commonly dominant $\mathrm{H}_{2} \mathrm{O}$ abundance in interstellar ice mantles leads to $\mathrm{OH}^{\bullet}$ radical formation upon irradiation and those radicals are incorporated into the structure of organic refractory residue species.

ii) Evidence of this is the mentioned above presence of HMT in organic refractory residues, a cage structure molecule, especially when $\mathrm{CH}_{3} \mathrm{OH}$ is one of the starting ice components.

iii) The $3.4 \mu \mathrm{m}$ subfeatures in organic refractory residue spectra are shifted to higher frequencies compared to the $-\mathrm{CH}_{2}-$ str. modes of polyethylene $\left(-\mathrm{CH}_{2}-\right)_{n}$ at 2917 and $2855 \mathrm{~cm}^{-1}$. That shift in frequency and the width of the subfeatures suggests that functional groups, like hydroxy $(\mathrm{OH})$, carbonyl $(\mathrm{C}=\mathrm{O})$ and other electrophylic groups observed in the organic refractory residue spectra, interact with the adjacent $\mathrm{CH}_{2}$ bonds that constitute organic refractory residues, supporting this option. As mentioned by Jenniskens et al. (1993), according to Wexler (1967) the intensity of the $\mathrm{C}-\mathrm{H}$ stretch is significantly reduced if functional groups like $\mathrm{C}=\mathrm{O}$ and $\mathrm{OH}$ are adjacent, resulting in the relatively weak $3.4 \mu \mathrm{m}$ feature of most organic refractory residue spectra. In addition, many molecules detected by gas chromatography coupled to mass spectrometry or inferred from infrared spectra of organic refractory residues are of the $\mathrm{R}-\left(\mathrm{CH}_{2}\right)_{n}$ $\mathrm{R}^{\prime}$ type, such as alcohols, carboxylic acids and their ammonium salts, amides, amines, and polyoxymethylene-like species (Agarwal et al. 1985; Briggs et al. 1992; Bernstein et al. 1995; Muñoz Caro \& Schutte 2003).

Among the different options given above, that is i) the aliphatic chains are long and poorly branched; ii) cyclic or cage molecules with $\mathrm{CH}_{2}$ and no $\mathrm{CH}_{3}$ groups; and iii) chains of the type $\mathrm{R}-\left(\mathrm{CH}_{2}\right)_{n}-\mathrm{R}^{\prime}$ where $n$ does not need to be a large number because there are no $-\mathrm{CH}_{3}$ bonds in the molecular structure, the main contribution to the $3.4 \mu \mathrm{m}$ feature of organic refractory residues is thus given by species of the iii) type. Most $\mathrm{CH}_{n}$ bonds in the organic refractory residue molecules reported in Table I of Briggs et al. (1992), resulting from GC-MS analysis of an organic refractory residue made from $\mathrm{H}_{2} \mathrm{O}: \mathrm{NH}_{3}: \mathrm{CO}=5: 5: 1$ ice, are $\mathrm{CH}_{2}$ bonds and there are no $\mathrm{CH}_{3}$ bonds, in accordance with the predominance of $\mathrm{CH}_{2}$ stretchings in the $3.4 \mu \mathrm{m}$ feature profile of organic refractory residues (Fig. 1). In addition, there are in total 3.4 times more $\mathrm{OH}$ than $\mathrm{COOH}$ groups, the two most abundant functional groups in Table I of Briggs et al. (1992); furthermore, the intrinsic intensity of $\mathrm{CH}_{3}$, and presumably also $\mathrm{CH}_{2}$ groups, adjacent to $\mathrm{OH}$ groups is reduced by about 3 times, compared to a 7-9 times reduction when adjacent to carbonyl $\mathrm{C}=\mathrm{O}$ groups (Wexler 1967) like the carboxy carbonyl group in the $-\mathrm{COOH}$ termination of carboxylic acids. That means that for the organic refractory residue molecules reported by Briggs et al. (1992), $\mathrm{CH}_{2}$ bonds adjacent to $\mathrm{OH}$ groups

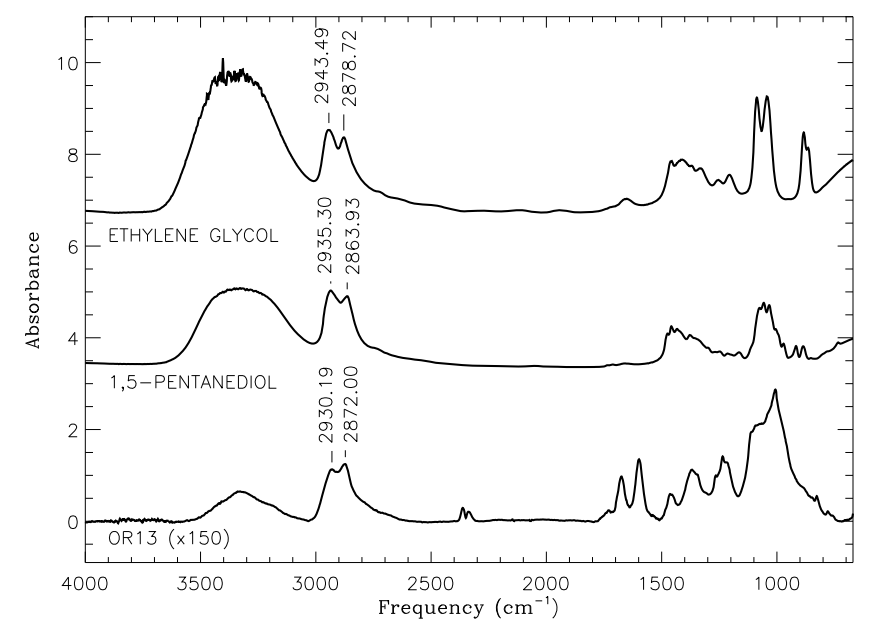

Fig. 3. Comparison between OR13 spectrum (bottom trace) and two alcohols, 1,5-pentanediol (middle trace) and ethylene glycol (top trace). The spectra were obtained at room temperature.

contribute roughly 9 times more to the intensity of the $3.4 \mu \mathrm{m}$ feature than $\mathrm{CH}_{2}$ bonds adjacent to $\mathrm{C}=\mathrm{O}$ groups. Therefore, for the organic refractory residue molecules of Briggs et al. (1992), the $3.4 \mu \mathrm{m}$ feature intensity and profile result mainly from the stretching modes of $\mathrm{CH}_{2}$ groups adjacent to hydroxy $\mathrm{OH}$ groups.

The above conclusion was double checked by comparison of the $3.4 \mu \mathrm{m}$ feature of organic refractory residues to that of alcohols with no $\mathrm{CH}_{3}$ groups. Figure 3 compares the spectra of 1,5-pentanediol ( $\mathrm{HO}-\left(\mathrm{CH}_{2}\right)_{5}-\mathrm{OH}$, middle trace) and ethylene glycol $\left(\mathrm{HO}-\left(\mathrm{CH}_{2}\right)_{2}-\mathrm{OH}\right.$, top trace) to the spectrum of OR13 (bottom trace). It shows that the $3.4 \mu \mathrm{m}$ feature profile of organic refractory residues resembles that of molecules of the above iii) type, of the form $\mathrm{R}-\left(\mathrm{CH}_{2}\right)_{n}-\mathrm{R}^{\prime}$ where $\mathrm{R}=\mathrm{R}^{\prime}=\mathrm{OH}$, and the other alcohol absorptions in the mid-infrared can be accomodated in the organic refractory residue spectrum shown. If $\mathrm{R}=\mathrm{R}^{\prime}=\mathrm{COOH}$ the fit to the $3.4 \mu \mathrm{m}$ feature profile of organic refractory residues is not as good and, furthermore, the intrinsically strong $\mathrm{C}=\mathrm{O}$ str. intensity of carboxylic acids is weak or absent in many organic refractory residue spectra, indicating a smaller contribution of $\mathrm{C}=\mathrm{O}$ relative to $\mathrm{OH}$ groups. Even the carboxylic acids in the organic refractory residues reported in Table I of Briggs et al. (1992), with glycolic acid $\left(\mathrm{HOCH}_{2} \mathrm{COOH}\right)$ the most abundant, contain one or two $\mathrm{OH}$ adjacent to $\mathrm{CH}_{2}$ groups, except the low-abundance oxamic acid $\left(\mathrm{NH}_{2} \mathrm{COCOOH}\right)$. These carboxylic acids are mainly in the form of ammonium salts of carboxylic acids in the organic refractory residue samples containing $\mathrm{N}$, i.e. made from ice mixtures with a $\mathrm{NH}_{3}$ component, prior to the solution of organic refractory residues required for gas chromatographic analysis. The $\mathrm{CH}_{2}-\mathrm{OH}$ groups in such salts contribute considerably to the $3.4 \mu \mathrm{m}$ feature of organic refractory residues (Muñoz Caro \& Schutte 2003, see Fig. 4 of that paper).

We mentioned above that the $3.4 \mu \mathrm{m}$ features of organic refractory residue spectra, shown in Fig. 1, resemble the same feature in the 1,5-pentanediol spectrum, shown in Fig. 3. In general, subtraction of both features after scaling shows a small excess in the blue wing of the $3.4 \mu \mathrm{m}$ feature of the organic refractory residue spectra compared to the same feature in the 1,5-pentanediol spectrum. This excess could be due to the asymmetric stretching mode of $\mathrm{CH}_{3}$ bonds around $2960 \mathrm{~cm}^{-1}$, since 1,5-pentanediol lacks $\mathrm{CH}_{3}$ groups. Integration of the $3.4 \mu \mathrm{m}$ feature of organic refractory residue spectra, and integration of the 

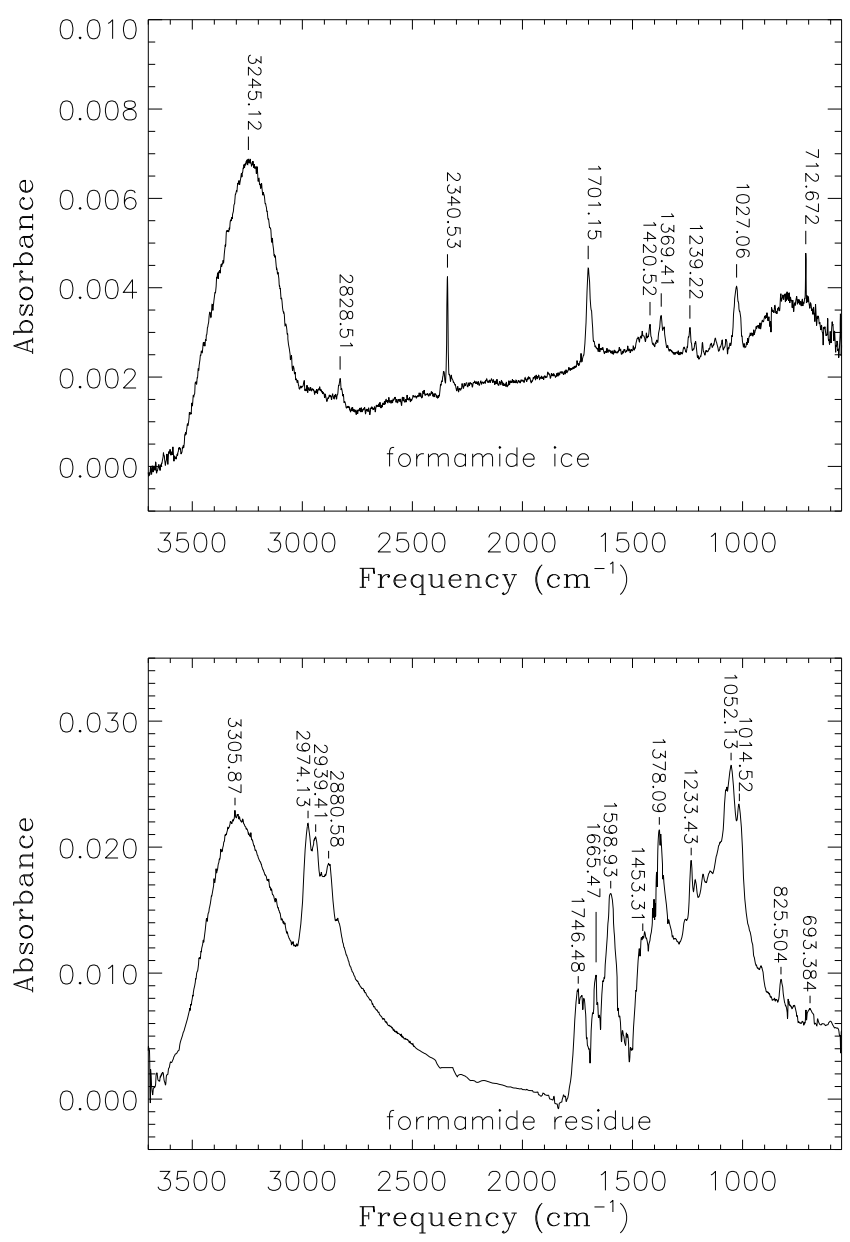

Fig. 4. Top: spectrum corresponding to deposition of formamide ice. Bottom: spectrum of residue made by simultaneous deposition and UV-irradiation of formamide, followed by warm-up to room temperature.

mentioned above excess on the blue wing, was performed, using the formula

$N=\int_{\text {band }} \frac{\tau_{\nu} \mathrm{d} v}{A}$

where $N$ is the column density in $\mathrm{cm}^{-2}, \tau$ is the optical depth of the band, $\mathrm{d} v$ is the wavenumber differential in $\mathrm{cm}^{-1}$, and $A$ is the band strength in $\mathrm{cm} \mathrm{molec}^{-1}$. We adopted the band strengths for hexane ice (Dartois et al. 2004a), $A\left(\mathrm{CH}_{3}\right)=1.25 \times 10^{-17} \mathrm{~cm}$ $(\mathrm{C} \text { atom })^{-1}$ and $A\left(\mathrm{CH}_{2}\right)=8.4 \times 10^{-18} \mathrm{~cm}(\mathrm{C} \text { atom })^{-1}$, and obtained that $\mathrm{N}\left(\mathrm{CH}_{3}\right) / \mathrm{N}\left(\mathrm{CH}_{2}\right) \leq 0.1$. This value is an upper limit that should be regarded with caution, because the $\mathrm{CH}$ stretching modes are perturbed by the neighboring electrophilic groups, and the subfeatures due to the $\mathrm{CH}_{3}$ stretchings are blended with those due to the $\mathrm{CH}_{2}$ stretchings.

It is concluded that for organic refractory residues made from irradiation of realistic interstellar ice analogs, the main contribution to the $3.4 \mu \mathrm{m}$ feature corresponds to the symmetric and asymmetric $\mathrm{CH}_{2}$ stretching modes perturbated by adjacent hydroxyl OH groups.

\subsection{Residues produced by "exotic" ice species compared to astrophysically relevant organic refractory residues}

Muñoz Caro \& Schutte (2003) found that the relative abundances of the irradiation products present in organic refractory
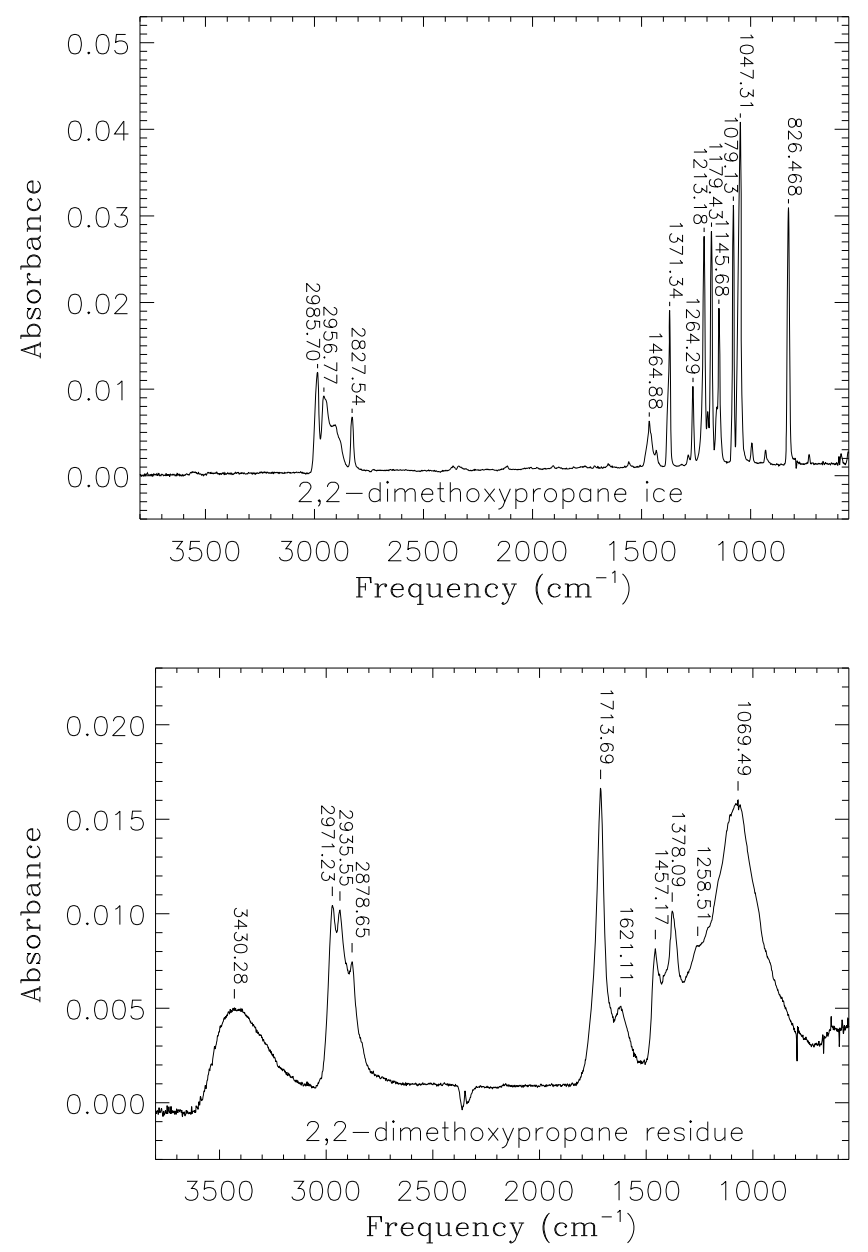

Fig. 5. Top: spectrum corresponding to deposition of 2,2dimethoxypropane ice. Bottom: spectrum of residue made by simultaneous deposition and UV-irradiation of 2,2-dimethoxypropane, followed by warm-up to room temperature. The band between $2400-2300 \mathrm{~cm}^{-1}$ is $\mathrm{CO}_{2}$ gas absorbing along the beam of the infrared spectrometer.

residues vary considerably as a function of the starting ice composition, UV dose, and frequency of the UV photons. Despite that, here we showed that those organic refractory residues share a similar $3.4 \mu \mathrm{m}$ feature profile. An important question is thus whether the broad double-peaked $3.4 \mu \mathrm{m}$ feature profile of organic refractory residues can be reproduced by UV-irradiation of "exotic" ice components, i.e. non-realistic analogs of interstellar and circumstellar ice mantles. That depends on whether there are strong memory effects on the synthesis of irradiation products as a function of the different composition of the starting ice, in other words if UV-irradiation can fragment the ice precursors sufficiently to fully erase the initial composition of the starting ice so that two different ices with the same elemental atomic composition would lead to the same residue after irradiation. For that purpose, pure formamide $\mathrm{CHONH}_{2}$ and 2,2-dimethoxypropane $\left(\mathrm{CH}_{3}\right)_{2} \mathrm{C}\left(\mathrm{OCH}_{3}\right)_{2}$ ices were UV-irradiated under the same conditions as interstellar ice analog mixtures. Their ice spectra are shown on top panels of Figs. 4 and 5. The residue spectrum of formamide in Fig. 4, bottom panel, is similar to that of pure methanol, and thus shows a $3.4 \mu \mathrm{m}$ feature composed of subfeatures associated with $\mathrm{CH}_{3}$ and $\mathrm{CH}_{2}$ stretchings, different to the $3.4 \mu \mathrm{m}$ feature of organic refractory residues. The same holds for the $3.4 \mu \mathrm{m}$ feature of the 2,2-dimethoxypropane residue in 
Fig. 5, bottom panel; the spectrum shows prominent bands due to functional groups at $3430(\mathrm{OH}$ str. $), 1714(\mathrm{C}=\mathrm{O}$ str. in saturated aliphatic ketones), and $1069 \mathrm{~cm}^{-1}$ (C-OH str. in alcohols). As a result, it was found that the $3.4 \mu \mathrm{m}$ feature of organic refractory residues was not reproduced when formamide or 2,2-dimethoxypropane, two $\mathrm{OH}$-free and non-realistic analogs of interstellar/circumstellar ices, were UV-irradiated.

\section{Astrophysical implications}

This work shows that the common characteristic broad doublepeaked $3.4 \mu \mathrm{m}$ feature profile of organic refractory residues, with subfeatures at $\sim 2926 \mathrm{~cm}^{-1}(3.42 \mu \mathrm{m})$ and $2876 \mathrm{~cm}^{-1}(3.48 \mu \mathrm{m})$, can be used as a tracer of refractory organic matter made from irradiation and subsequent sublimation of interstellar and circumstellar ice mantles, and this refractory organic matter might be observed on grains that were not further processed after sublimation of the ice. The $3.4 \mu \mathrm{m}$ feature of organic refractory residues is a good tracer for several reasons: a) it has subfeatures that translate into a more specific band profile when compared to the bands attributed to functional groups in organic matter; b) bands due to functional groups in organic refractory residues can overlap with ice bands, or bands due to hydrated silicates or carbonates in grains, but the $3.4 \mu \mathrm{m}$ feature of organic refractory residues could still be observed superposed on the red wing of water ice absorption; c) often the only observing infrared window available is the one corresponding to this band. In a recent publication, we searched for solid $\mathrm{O}$ - and N-rich organic matter of prebiotic interest in space that relied on the infrared and Raman spectroscopic comparison of organic refractory residues to diffuse interstellar medium carbon, and the carbon fraction in comets, meteorites and IDPs. It was found that comets are expected to be the main source of such organic matter for the early earth (Muñoz Caro \& Dartois 2007). The results presented here validate previous publications where the $3.4 \mu \mathrm{m}$ feature of organic refractory residues was compared to that found in different space environments, which main conclusions are summarized below.

Figure 6 shows a comparison of the $3.4 \mu \mathrm{m}$ organic refractory residue feature to those of IDP L2036-R9, the carbon matrix of the Orgueil carbonaceous chondrite after hydrolysis, and the one observed toward GC IRS8 as a probe of the diffuse interstellar medium. Clearly, it shows that the $3.4 \mu \mathrm{m}$ feature of organic refractory residues differs from that of hydrocarbon polymers that are poor in $\mathrm{O}$ and $\mathrm{N}$, like the hydrogenated amorphous carbon, a-C:H (e.g. Sandford et al. 1991; Duley et al. 1998; Mennella et al. 1999; Dartois et al. 2004b, 2005), present in carbon grains of the diffuse interstellar medium (as reported by Pendleton \& Allamandola 2002), carbonaceous chondrites like Orgueil, and IDPs (Muñoz Caro et al. 2006). The mid-infrared fingerprints of organic refractory residues are also different from those present in the spectra of IDPs, carbonaceous chondrites and diffuse medium carbon grains (Muñoz Caro et al. 2006; Dartois et al. 2005). This indicates that the chemical structure and composition of organic refractory residues is different to the a-C:H materials present in IDPs, carbonaceous chondrites, and diffuse medium carbon grains (Muñoz Caro et al. 2006; Muñoz Caro \& Dartois 2007). Nevertheless, a fraction of the organic refractory residue matter, dating from the local dense cloud or the solar nebula, could still be preserved in cometary nuclei that did not experience heating at temperatures above $300{ }^{\circ} \mathrm{C}$, and could be the precursor of the $\mathrm{D}$ and ${ }^{15} \mathrm{~N}$-enriched carbon component of IDPs and carbonaceous chondrites (Muñoz Caro et al. 2006; Muñoz Caro \& Martínez-Frías 2007). The $3.4 \mu \mathrm{m}$

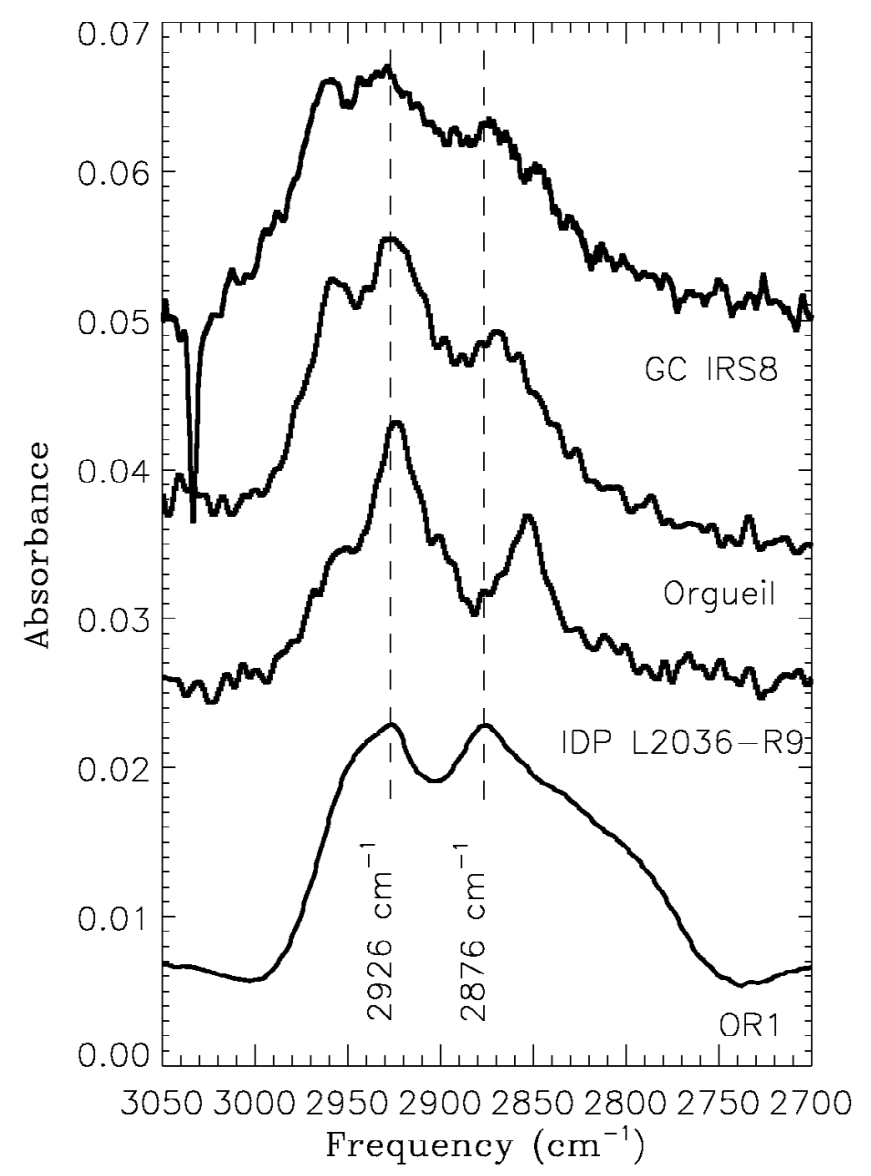

Fig. 6. Comparison of the infrared $3.4 \mu \mathrm{m}$ features corresponding to an organic refractory residue (OR1, see Sect. 3.1), an IDP named L2036-R9, the Orgueil residue obtained from hydrolysis, and the Galactic Center source GC IRS8.

feature of organic refractory residues was also used recently for comparison to the carbon bulk in cometary grains collected by Stardust (Muñoz Caro et al. 2008), and found to be very different. IDPs collected prior to atmospheric entry heating and cometary grains that were not annealed at temperatures higher than $300{ }^{\circ} \mathrm{C}$ during capture might be richer in organic matter rich in $\mathrm{O}$ and $\mathrm{N}$. The proportion of organic matter of prebiotic interest over the total carbon content in comets, meteorites and IDPs is a key parameter to constrain the contribution of extraterrestrial delivery to the origin of life on Earth.

This paper could serve to promote the search for solid organic matter made from ice photoprocessing followed by warmup toward hot cores, regions around YSOs, and solar system bodies like comets. Infrared observations toward a few YSOs, like IRAS $04579+4703$, show a $3.4 \mu \mathrm{m}$ feature that is compatible with that of organic refractory residues (Ishii et al. 2002), but the resolution is still too poor for a proper comparison, and improved observations with higher resolution and signal-to-noise ratio are required. The same line of sight can intersect both circumstellar and diffuse interstellar carbon grains. Therefore, to assess the presence of organic refractory residue matter in circumstellar regions, the detected $3.4 \mu \mathrm{m}$ feature profile should differ significantly from that detected toward diffuse medium lines of sight. By adding the $3.4 \mu \mathrm{m}$ feature, attributed to diffuse interstellar carbon grains (top trace of Fig. 6), to the same feature of organic refractory residues (bottom trace of Fig. 6), we found that the $3.4 \mu \mathrm{m}$ feature of organic refractory matter is clearly detected when its optical depth value is equal to or larger than the optical 
depth value of the $3.4 \mu \mathrm{m}$ feature of diffuse interstellar carbon grains.

\section{Conclusions}

The $3.4 \mu \mathrm{m}$ feature of organic refractory residues has a characteristic band shape, consisting of two subfeatures around $2925 \mathrm{~cm}^{-1}(3.42 \mu \mathrm{m})$ and $2875 \mathrm{~cm}^{-1}(3.48 \mu \mathrm{m})$, that is not very sensitive to changes in the composition of the starting ice mixture. This feature, however, was not reproduced in irradiation experiments using non-realistic $\mathrm{OH}$-free analogs of interstellar and circumstellar ice mantles; the reason for this is that the $3.4 \mu \mathrm{m}$ feature of organic refractory residues is mainly attributed to the interaction of $\mathrm{OH}$, and to a lesser extent other electrophylic groups, with the adjacent $\mathrm{CH}_{2}$ bonds that constitute organic refractory residues.

This band was used for comparison of organic refractory residues to the carbon in diffuse interstellar grains (Pendleton \& Allamandola 2002), or in solar system objects like comets and IDPs (Muñoz Caro et al. 2006, 2008), and here it was compared to the carbon fraction of the Orgueil carbonaceous chondrite; the $3.4 \mu \mathrm{m}$ bands in the spectra of all these materials are different to the $3.4 \mu \mathrm{m}$ band in the spectra of organic refractory residues. Indeed, many IDPs, carbonaceous chondrites like Orgueil and Murchison, and the infrared bands associated with diffuse medium carbon grains, contain different forms of hydrogenated amorphous carbon, a hydrocarbon polymer material poor in $\mathrm{O}$ and $\mathrm{N}$, compared to the products of ice UV-irradiation that compose organic refractory residues (see Muñoz Caro \& Dartois 2007, for a review). So far, no material from space has been found that produces a $3.4 \mu \mathrm{m}$ feature with the same profile as the one present in the organic refractory residue spectra. Even so, IDPs collected prior to atmospheric entry heating, comets, and even some meteorites, might preserve organic matter rich in $\mathrm{O}$ and $\mathrm{N}$ made from ice photoprocessing. Organic globules, enriched in $\mathrm{D}$ and ${ }^{15} \mathrm{~N}$, were detected in the Tagish Lake meteorite; these globules probably originated as organic ice coatings on preexisting grains that were photochemically processed into organic refractory matter (Nakamura-Messenger et al. 2006). Using the $3.4 \mu \mathrm{m}$ feature of organic refractory residues as a tracer, we propose new observations in the infrared, with improved resolution and signal-to-noise ratio, to probe the presence of organic matter made by ice UV-irradiation and warmup, toward hot cores, regions around YSOs, and icy bodies in the solar system.

Acknowledgements. We thank Willem Schutte for his guidance and support. We thank Michel Nuevo for kindly providing an infrared spectrum. G.M.M.C. was supported by a Marie Curie Individual Fellowship from the European Union and a Ramón y Cajal research contract from the MCYT in Spain.

\section{References}

Allamandola, L. J., Sandford, S. A., \& Valero, G. J. 1988, Icarus, 76, 225 Agarwal, V. K., Schutte, W. A., Greenberg, J. M., et al. 1985, Origins of Life and Evolution of the Biosphere, 16, 21

Bernstein, M. P., Sandford, S. A., Allamandola, L. J., \& Chang, S. 1994, J. Phys. Chem., 98, 12206

Bernstein, M. P., Sandford, S. A., Allamandola, L. J., Chang, S., \& Scharberg, M. A. 1995, ApJ, 454, 327

Bernstein, M. P., Dworkin, J. P., Sandford, S. A., Cooper, G. W., \& Allamandola, L. J. 2002, Nature, 416, 401

Briggs, R., Ertem, G., Ferris, J. P., et al. 1992, Origins of Life and Evolution of the Biosphere, 22, 287

Dartois, E., Demyk, K., d'Hendecourt, L., \& Ehrenfreund, P. 1999, A\&A, 351, 1066

Dartois, E., Marco, O., Muñoz Caro, G. M., et al. 2004a, A\&A, 423, 549

Dartois, E., Muñoz Caro, G. M., Deboffle, D., \& d'Hendecourt, L. 2004b, A\&A, 423, L33

Dartois, E., Muñoz Caro, G. M., Deboffle, D., Montagnac, G., \& d'Hendecourt, L. 2005, A\&A, 432, 895

Duley, W. W., Scott, A. D., Seahra, S., \& Dadswell, G. 1998, ApJ, 503, L183

Ehrenfreund, P., Kerkhof, O., Schutte, W. A., et al. 1999, A\&A, 350, 240

Gerakines, P. A., Whittet, D. C. B., Ehrenfreund, P., et al. 1999, ApJ, 522, 357

Gibb, E. L., Whittet, D. C. B., \& Chiar, J. E. 2001, ApJ, 558, 702

Ishii, M., Nagata, T., Chrysostomou, A., \& Hough, J. H. 2002, AJ, 124, 2790

Jenniskens, P., Baratta, G. A., Kouchi, A., et al. 1993, A\&A, 273, 583

Meierhenrich, U. J., Muñoz Caro, G. M., Schutte, W. A., et al. 2005, Chem. Eur. J., 11, 4895

Mennella, V., Brucato, J. R., Colangeli, L., \& Palumbo, P. 1999, ApJ, 524, L71 Muñoz Caro, G. M., \& Dartois, E. 2007, in Planetary Science, ed. W.-H. Ip, \& A. Bhardwaj (World Scientific), Adv. in Geosciences, 7, 155

Muñoz Caro, G. M., \& Schutte, W. A. 2003, A\&A, 412, 121

Muñoz Caro, G. M., \& Martínez-Frías, J. 2007, in Proceedings of Dust in Planetary Systems, Kauai, Hawaii, USA, ESA SP-643, 133

Muñoz Caro, G. M., Meierhenrich, U. J., Schutte, W. A., et al. 2002, Nature, 416, 403

Muñoz Caro, G. M., Meierhenrich, U. J., Schutte, W. A., Thiemann, W. H.-P., \& Greenberg, J. M. 2004, A\&A, 413, 209

Muñoz Caro, G. M., Matrajt, G., Dartois, E., et al. 2006, A\&A, 459, 147

Muñoz Caro, G. M., Dartois, E., \& Nakamura-Messenger, K. 2008, A\&A, 485, 743

Nakamura-Messenger, K., Messenger, S., Keller, L. P., Clemett, S. J., \& Zolensky, M. E. 2006, Science, 314 (5804), 1439

Nuevo, M., Meierhenrich, U. J., Muñoz Caro, G. M., et al. 2006, A\&A, 457, 741 Pendleton, Y. J., \& Allamandola, L. J. 2002, ApJS, 138, 75

Pontoppidan, K. M., Dullemond, C. P., van Dishoeck, E. F., et al. 2005, ApJ, 622,463

Sandford, S. A., Allamandola, L. J., Tielens, A. G. G. M., et al. 1991, ApJ, 371, 607

Schutte, W. A., \& Khanna, R. K. 2003, A\&A, 398, 1049

Schutte, W. A., Allamandola, L. J., \& Sandford, S. A. 1993, Icarus, 104, 118

Shen, C. J., Greenberg, J. M., Schutte, W. A., \& van Dishoeck, E. F. 2004, 415, 203

Thi, W. F., Pontoppidan, K. M., van Dishoeck, E. F., Dartois, E., \& d'Hendecourt, L. 2002, A\&A, 394, L27

Weber, P., \& Greenberg, J. M. 1985, Nature, 316, 403

Wexler, A. S. 1967, Appl. Spectrosc. Rev., 1, 29 